\title{
Clinical outcome after total knee replacement
}

\author{
Roland Becker ${ }^{1} \cdot$ Reha N. Tandogan ${ }^{2} \cdot$ Jon Karlsson $^{3}$
}

Published online: 17 May 2015

(C) European Society of Sports Traumatology, Knee Surgery, Arthroscopy (ESSKA) 2015

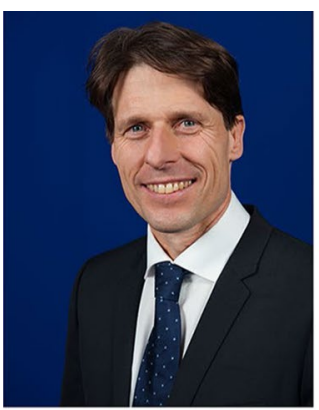

Roland Becker

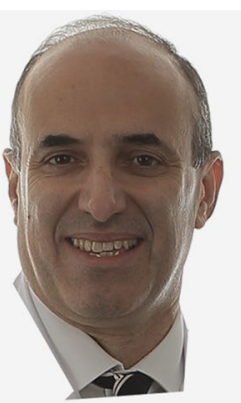

Reha N. Tandogan

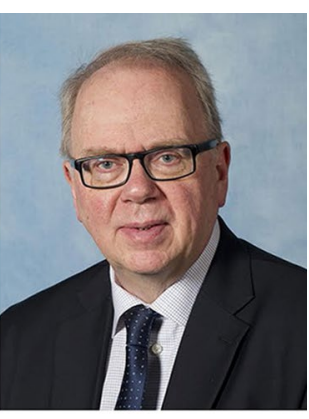

Jon Karlsson
The majority of studies in the field of total knee arthroplasty (TKA) analyses patients' clinical outcome and function. The current issue provides an update on clinical outcome taking different aspects into consideration. These studies are very important in order to learn more about patients' satisfaction and functional performance after joint replacement. Findings will help in identifying the most relevant aspects for successful TKA. The

Roland Becker

roland_becker@yahoo.de

Reha N. Tandogan

rtandogan@ ortoklinik.com; rtandogan@gmail.com

Jon Karlsson

jon.karlsson@telia.com

1 Department of Orthopaedic and Traumatology, City Hospital Brandenburg, Hochstrasse 29, 14770 Havel, Brandenburg, Germany

2 Cankaya Orthopedics, Bulten Sokak No. 44, Kavaklidere, Ankara, Turkey

3 Department of Orthopaedics, Sahlgrenska University Hospital, 43180 Mölndal, Sweden concept of resurfacing the distal femur and proximal tibia has not changed much, and the majority of implants is still placed perpendicular to the mechanical axis. However, numerous designs have been developed over the last 40 years with the aim of achieving more natural knee kinematics.

How much does the implant design affect our clinical outcome? Should we use, for instance, a fixed or a mobile design? The prospectively designed study published in the current issue did not show any difference in clinical outcome between the two designs according to the Oxford Knee Score, American Knee Society Score, Short Form-12 and range of motion [3]. The finding is in line with the Cochrane Review published earlier this year [7]. The authors of the Cochrane Review also stated that there is still a lack of evidence because many of these studies are rather of low or moderate quality. Clinical research is very demanding for different reasons. First, the study needs to be very well designed in order to be able to answer the question of interest, which should be expressed in the author's hypothesis. Secondly, patients have to be selected and followed up for a long time. This requires good compliance by the patients. Some of them may drop out, which 
may compromise such designed study. Clinical studies also require staff. Taking all these aspects into consideration, clinical studies are time consuming and expensive.

Here is another example of an ongoing debate. It is about the usage of the posterior-stabilised (PS) and cruciateretaining (CR) design. The protagonists for the PS design claim better soft tissue balancing and range of motion compared with the CR design. On the other hand, the posterior cruciate ligament is important for proprioception and knee kinematics. A meta-analysis revealed a slightly better range of motion and flexion in favour of the PS design [4]. However, the clinical outcome did not show significant difference [9]. It is not very surprising that there is no difference between both the implant designs because gait analysis also did not show any difference in gait pattern [12]. In conclusion, the surgeon's preference might be the choice for the implant design.

The function of the standard and the high flex design has been reviewed in the literature, and an increase in flexion of $2^{\circ}$ was found with the high flexion design [1]. Do we need the high flexion design in TKA, when the increase in flexion is only very small and clinical relevance still doubtful?

Summarising the data after comparing the fixed and mobile bearing design, the CR and PS design and the standard and high flex design, there does not seem to be much difference in function and clinical outcome. However, one may presume that the current tools for patients' evaluation may not be sensitive enough for the detection of clinical differences between the designs. On the other hand, one may presume that the implantation of a total joint causes such a tremendous change in knee kinematics, soft tissue tension and function that differences in component design can be neglected. Patient's evaluation is based on outcome scores and range of motion in general. There is no doubt that these data are important and need to be evaluated.

When we evaluate the outcome of our patients, we look rather in a more general perspective and most of the studies are designed retrospectively. There might be some patients, who may show more improvement when using one or the other design with respect to their activity for instance. Registries and retrospective studies are unable to provide an answer. Do we need a more individual approach to our patients?

The soft tissue around the knee seems to be the structure of major interest. Most of the sensation around the knee comes from the medial and lateral retinaculae and may be the cause of pain [5]. Preserving or resection of the PCL does not affect proprioception, muscle strength or clinical outcome after TKA, but good function of the collateral ligaments is required [11]. Aunan et al. [2] measured the mediolateral laxity after TKA. They reported on a negative correlation between increased medial laxity in neutral and varus aligned knees and the clinical outcome. As stated by the authors, it remains questionable how much mediolateral joint laxity should be considered as optimal. In contrast, the results by Okazaki et al. [10] showed no correlation between the femorotibial opening in varus and valgus and patients outcome. Looking into more detail, the studies are not comparable. While Aunan et al. used spatulas, which were manually inserted into the joint space in order to measure mediolateral laxity in $0^{\circ}$ and $90^{\circ}$ of flexion, Okazaki et al. used the Telos device at $150 \mathrm{~N}$ for laxity measurement at $10^{\circ}$ of knee flexion. This is an example showing opposite results, but more detailed information is required in order to understand that findings are not comparable. The knowledge about the material and methods of studies are crucial for understanding results. However, intraoperative soft tissue management seems to be the most demanding and crucial part in TKA to achieve good functional and clinical outcome.

The condition prior to surgery should not be neglected when the outcome after TKA is discussed. The study by Ishida et al. [8] showed that lag of extension and varus deformity prior to surgery correlates negatively with the outcome after surgery.

The preoperative range of motion is also a strong predictor for maximal knee flexion [6].

Patient evaluation is complex, and most of the time a specific aspect is moved into our focus. However, many other aspects are neglected at the same time, but these aspects may also have a tremendous input in knee function.

The complexity of the evaluation of patient's outcome makes clinical studies very demanding.

\section{References}

1. Arirachakaran A, Wande T, Pituckhanotai K et al (2015) Clinical outcomes after high-flex versus conventional total knee arthroplasty. Knee Surg Sports Traumatol Arthrosc. doi:10.1007/ s00167-015-3557-0

2. Aunan E, Kibsgård TJ, Diep LM et al (2014) Intraoperative ligament laxity influences functional outcome 1 year after total knee arthroplasty. Knee Surg Sports Traumatol Arthrosc. doi:10.1007/ s00167-014-3108-0

3. Bailey O, Ferguson K, Crawfurd E et al (2014) No clinical difference between fixed- and mobile-bearing cruciate-retaining total knee arthroplasty: a prospective randomized study. Knee Surg Sports Traumatol Arthrosc. doi:10.1007/s00167-014-2877-9

4. Bercik MJ, Joshi A, Parvizi J (2013) Posterior cruciate-retaining versus posterior-stabilized total knee arthroplasty: a meta-analysis. J Arthroplasty 28:439-444

5. Dye SF, Vaupel GL, Dye CC (1998) Conscious neurosensory mapping of the internal structures of the human knee without intraarticular anesthesia. Am J Sports Med 26:773-777

6. Gandhi R, de Beer J, Leone J et al (2006) Predictive risk factors for stiff knees in total knee arthroplasty. J Arthroplasty 21:46-52

7. Hofstede SN, Nouta KA, Jacobs W et al (2015) Mobile bearing vs fixed bearing prostheses for posterior cruciate retaining total knee arthroplasty for postoperative functional status in patients 
with osteoarthritis and rheumatoid arthritis. Cochrane Database Syst Rev 2:CD003130

8. Ishida K, Shibanuma N, Matsumoto T et al (2015) Factors affecting intraoperative kinematic patterns and flexion angles in navigated total knee arthroplasty. Knee Surg Sports Traumatol Arthrosc. doi:10.1007/s00167-015-3572-1

9. Li N, Tan Y, Deng Y et al (2014) Posterior cruciate-retaining versus posterior stabilized total knee arthroplasty: a meta-analysis of randomized controlled trials. Knee Surg Sports Traumatol Arthrosc 22:556-564
10. Okazaki K, Miura H, Matsuda S et al (2006) Asymmetry of mediolateral laxity of the normal knee. J Orthop Sci 11:264-266

11. Vandekerckhove PJ, Parys R, Tampere T et al (2014) Does cruciate retention primary total knee arthroplasty affect proprioception, strength and clinical outcome? Knee Surg Sports Traumatol Arthrosc. doi:10.1007/s00167-014-3384-8

12. van den Boom LG, Halbertsma JP, van Raaij JJ et al (2014) No difference in gait between posterior cruciate retention and the posterior stabilized design after total knee arthroplasty. Knee Surg Sports Traumatol Arthrosc 22:3135-3141 